\title{
Successful Treatment of Psychiatric Symptoms after Traumatic Brain Injury Using Deep Brain Stimulation to the Anterior Limb of Internal Capsule-Nucleus Accumbens
}

\author{
Bin Zhou', Weiping Kuang ${ }^{1}$, Hongxing Huang ${ }^{1}$, Yong Zhu', Xiaofeng Chen ${ }^{1}$, Liang $\mathrm{Li}^{2}$, Ping Yang ${ }^{1}$ \\ ${ }^{1}$ Department of Neurosurgery, Brain Hospital of Hunan Province, Clinical Medical School of Hunan University of Chinese Medicine, ${ }^{2}$ Provincial \\ Key Laboratory of TCM Diagnostics, Hunan University of Chinese Medicine, Changsha, Hunan Province, China
}

\begin{abstract}
Psychiatric symptoms are common after traumatic brain injury (TBI), and some patients have poor drug therapeutic efficacy. We report a successfully treated case of psychiatric symptoms after TBI using deep brain stimulation (DBS) to the anterior limb of internal capsule (ALIC)-nucleus accumbens (NAc) in a 76-year-old woman. The patient suffered from auditory hallucination, mood changes, and insomnia caused by TBI. Psychological test assessment showed the scores of Hamilton Anxiety Scale, Hamilton Depression Scale and Positive and Negative Syndrome Scale were 30 , 35, and 96 respectively. Head magnetic resonance imaging scan showed right temporal lobe encephalomalacia. Head magnetic resonance spectroscopy (MRS) showed bilateral basal ganglia choline increased relatively. After DBS to the ALIC-NAc, the target parameters were adjusted. The psychiatric symptoms were completely improved and the result of head MRS was normal in the end. The current report declares that DBS is reversible, adjustable and safe in the treatment of psychiatric symptoms caused by TBI. DBS to the ALIC-NAC should be considered as a possible treatment choice once a patient showed psychiatric symptoms after TBI.
\end{abstract}

KEY WORDS: Deep brain stimulation; Traumatic brain injury; Psychiatry; Internal capsule; Nucleus accumbens.

\section{INTRODUCTION}

The mechanism of psychiatric symptoms after traumatic brain injury (TBI) is complicated [1]. The function of the brain tissue will be reduced or disappear due to direct organic damage [2]. So many factors such as brain edema, intracranial hypertension and cerebral vasospasm will result in brain ischemia and hypoxia after TBI [3]. Especially, the temporal lobe, frontal lobe, hippocampus,

Received: May 2, 2019 / Revised: July 6, 2019

Accepted: July 29, 2019

Address for correspondence: Liang Li

Provincial Key Laboratory of TCM Diagnostics, Hunan University of Chinese Medicine, 300 Xueshi Road, Changsha 410208,

Hunan Province, China

E-mail: superliliang@126.com

ORCID: https://orcid.org/0000-0001-9994-4690

Ping Yang

Department of Neurosurgery, Brain Hospital of Hunan Province,

Clinical Medical School of Hunan University of Chinese

Medicine, 427 Middle Furong Road, Changsha 410007, Hunan

Province, China

E-mail: cuteping12384@126.com

ORCID: https://orcid.org/0000-0003-2375-0616 basal ganglion and limbic system which are related to mental activity are more sensitive to ischemia and hypoxia [4]. Their function and activities decrease gradually, and a series of psychiatric symptoms occur. Some researchers declared that antipsychotic drugs offered no satisfactory longterm benefit [5], which brought serious risks and burdens to society and families. Deep brain stimulation (DBS) has been reported repeatedly for refractory psychiatric treatment, such as attention deficit hyperactivity disorder [6] and obsessive-compulsive disorder [7]. However, there were no reports of DBS treatment on psychiatric symptoms after TBI. This study was the first time attempting to treat psychiatric symptoms after TBI using DBS to the anterior limb of internal capsule (ALIC)nucleus accumbens (NAc). After 18 months of follow-up, the patients' psychiatric symptoms had been alleviated significantly.

(c) This is an Open-Access article distributed under the terms of the Creative Commons Attribution Non-Commercial License (http://creativecommons.org/licenses/by-nc/4.0) which permits unrestricted non-commercial use, distribution, and reproduction in any medium, provided the original work is properly cited. 


\section{CASE}

A 76-year-old retired woman was sent to the local hospital after falling from a height of about 2 meters on January 15, 2015. The head computerized tomography showed subarachnoid hemorrhage, cerebral falx hernia and fracture of the occipital bone (Fig. 1). She suffered from severe psychiatric symptoms during hospitalization, such as auditory hallucination, insomnia, anxiety, poor mood and suicidal behavior. She always heard strange voice of men and women, felt her inner thoughts could be expressed by someone else in her ears. Wechsler Adult Intelligence Scale-Revised (China) was 104, and Minimum Mental State Examination was 29. She was referred to our hospital after receiving numerous antipsychotic treatments (olanzapine 5-15 mg/d + sertraline 50-100 $\mathrm{mg} / \mathrm{d}, \times 3$ month; quetiapine $50 \mathrm{mg}-0.4 \mathrm{~g} / \mathrm{d}+$ citalopram 5-20 mg/d, $\times 3$ month; aripiprazole 5-15 mg/d + mirta- zapine $7.5-15 \mathrm{mg} / \mathrm{d}, \times 2$ month; risperidone $1-4 \mathrm{mg} / \mathrm{d}$, $\times 2$ month; amisulpride $0.1-0.4 \mathrm{mg} / \mathrm{d}, \times 2$ month) without any long-lasting relief of symptoms for 1 year. The patient was informed of the risks and benefits of DBS and underwent extensive multidisciplinary evaluation before surgery to ensure her suitability and compatibility for the treatment. The presurgery scores of the Hamilton Anxiety Scale (HAMA) [8], Hamilton Depression Scale (HAMD) [9] and Positive and Negative Syndrome Scale (PANSS) [10] were 30, 35, and 96 respectively (Table 1). Head magnetic resonance imaging showed encephalomalacia at right temporal lobe (Fig. 2A). Head magnetic resonance spectroscopy (MRS) showed bilateral basal ganglia choline (Cho) level increased relatively (Fig. 3A, 3B).

DBS electrodes (L302; PinChi, Beijing, China) were implanted into ALIC and NAc, each with 4 contact points 3 $\mathrm{mm}$ long and separated from adjacent contacts by $4 \mathrm{~mm}$. Target coordinates for the electrode tip were $7 \mathrm{~mm}$ lateral
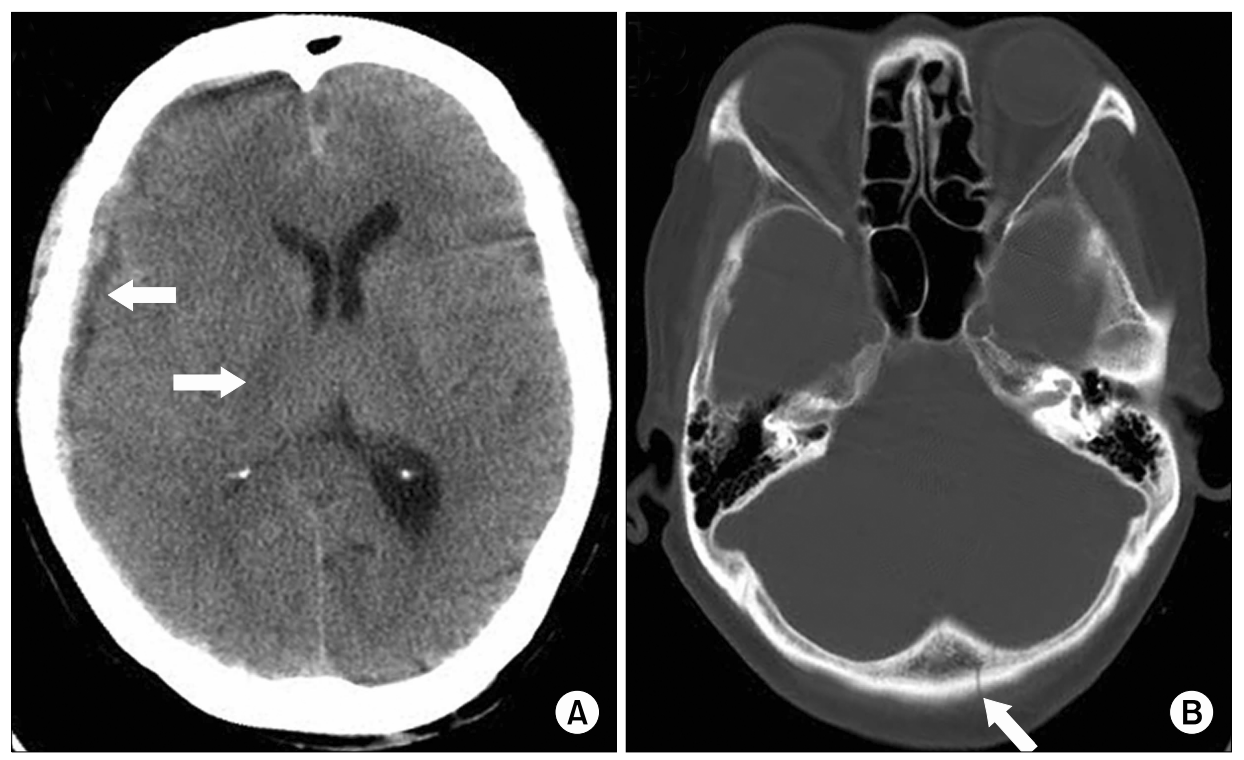

Fig. 1. Preoperative head computerized tomography (CT) images of the patient. Head CT showed mixed density shadows beneath the right frontal, temporal, parietal and occipital bones, with space-occupying lesions. A fracture line was visible on the occipital bone. Arrows represented subarachnoid hemorrhage, cerebral falx hernia $(A)$ and fracture of the occipital bone (B).

Table 1. Patient characteristics and stimulation settings

\begin{tabular}{|c|c|c|c|c|c|c|}
\hline Time & Stimulator settings & Left & Right & HAMA & HAMD & PANSS \\
\hline Preoperative & & & & 30 & 35 & 96 \\
\hline \multirow[t]{2}{*}{ Postoperative 1 month } & Points 1 (NAC) & $240 \mu \mathrm{s}, 150 \mathrm{~Hz}, 2.5 \mathrm{~V}$ & $240 \mu \mathrm{s}, 150 \mathrm{~Hz}, 2.5 \mathrm{~V}$ & & & \\
\hline & Points 3 (ALIC) & $240 \mu \mathrm{s}, 150 \mathrm{~Hz}, 2.5 \mathrm{~V}$ & $240 \mu \mathrm{s}, 150 \mathrm{~Hz}, 2.5 \mathrm{~V}$ & & & \\
\hline Postoperative 6 month & Points 1 & $240 \mu \mathrm{s}, 160 \mathrm{~Hz}, 3.0 \mathrm{~V}$ & $240 \mu \mathrm{s}, 160 \mathrm{~Hz}, 3.0 \mathrm{~V}$ & 11 & 10 & 40 \\
\hline Postoperative 8 month & Points 1 & $60 \mu \mathrm{s}, 90 \mathrm{~Hz}, 1.0 \mathrm{~V}$ & $60 \mu \mathrm{s}, 90 \mathrm{~Hz}, 1.0 \mathrm{~V}$ & & & \\
\hline Postoperative 12 month & Points 3 & $240 \mu \mathrm{s}, 160 \mathrm{~Hz}, 2.5 \mathrm{~V}$ & $240 \mu \mathrm{s}, 160 \mathrm{~Hz}, 2.5 \mathrm{~V}$ & 15 & 16 & 36 \\
\hline Postoperative 18 month & & & & 7 & 7 & 34 \\
\hline
\end{tabular}

NAc, nucleus accumbens; ALIC, anterior limb of internal capsule; HAMA, Hamilton Anxiety Scale; HAMD, Hamilton Depression Scale; PANSS, Positive and Negative Syndrome Scale. 

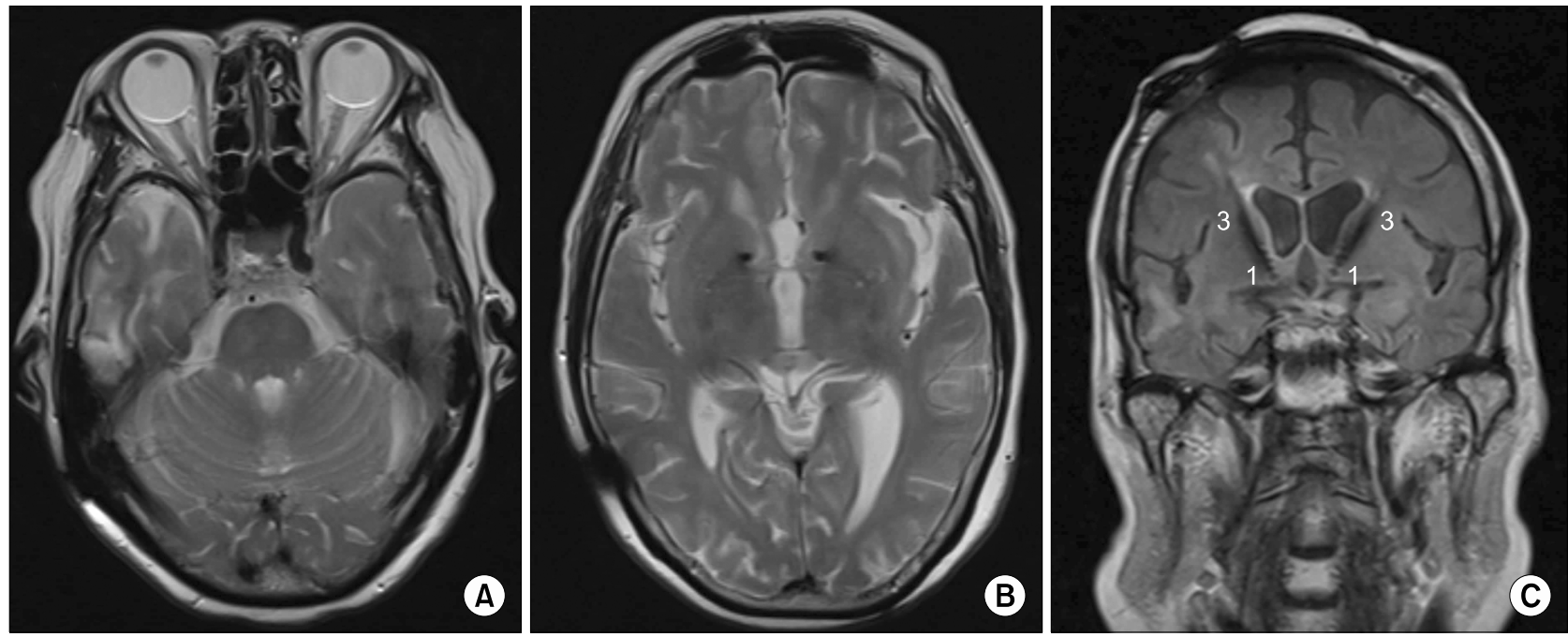

Fig. 2. Preoperative and postoperative head magnetic resonance images (MRI) of the patient. Axial (A) T2-weighted MRI indicated a focal lesion at the right temporal lobe with abnormally increased intensity, which was believed to be encephalomalacia preoperatively. Axial (B) and Coronal (C) showed the electrode trajectories through the anterior limb of internal capsule (ALIC) with the deepest electrode contact in the nucleus accumbens (NAc). 1 and 3 were contact points representing NAC and ALIC respectively.
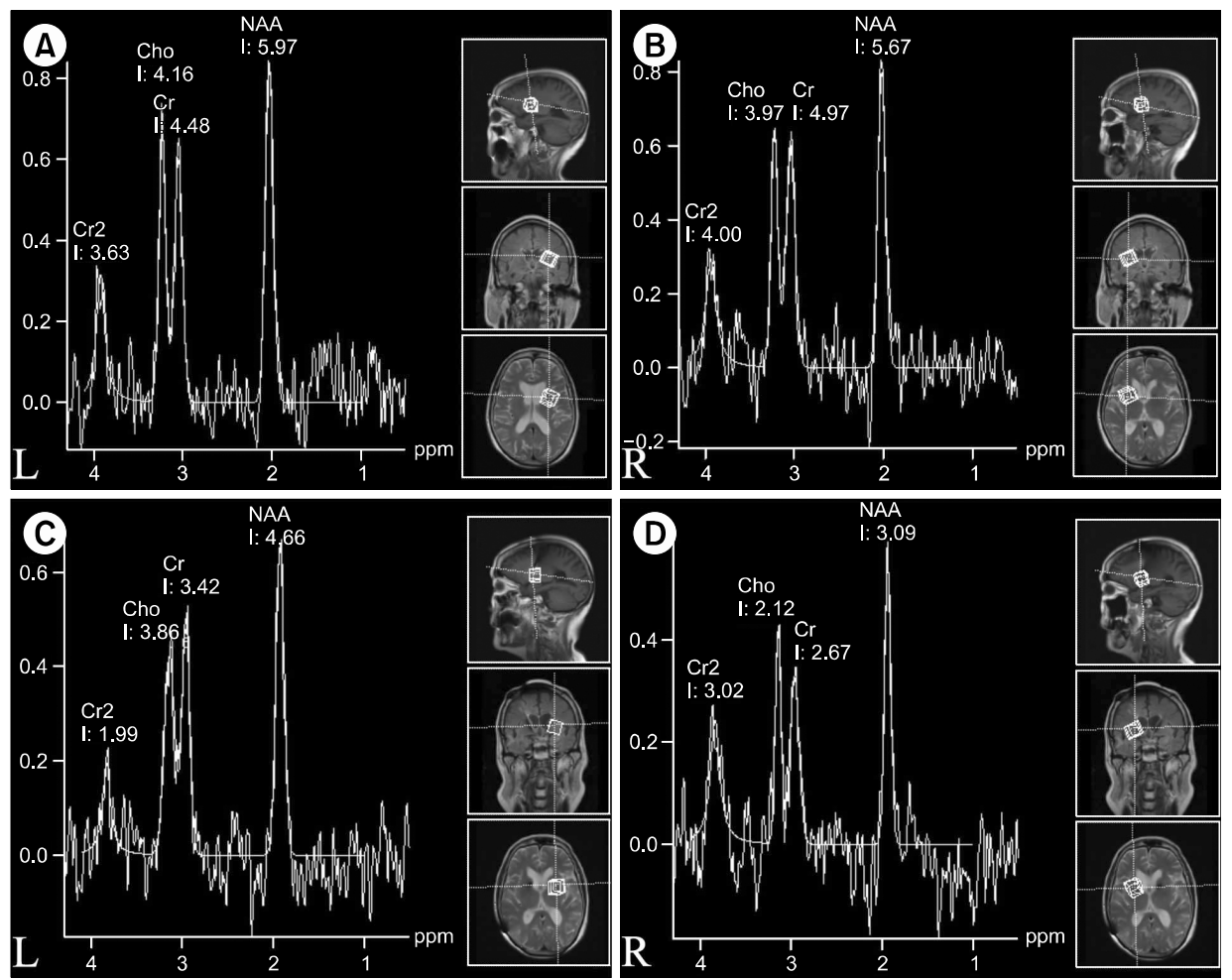

Fig. 3. Preoperative and postoperative head magnetic resonance spectroscopy (MRS) images of the patient. Head MRS showed preoperative (A, B) bilateral basal ganglia choline (Cho) level increased relatively, but levels of creatine $(\mathrm{Cr}), \mathrm{Cr} 2$, and $\mathrm{N}$-acetyl aspartate (NAA) were normal. Postoperative (C, D) levels of $\mathrm{Cho}, \mathrm{Cr}, \mathrm{Cr} 2$, and NAA were normal.

to the AC/PC line, $3 \mathrm{~mm}$ anterior to the anterior border of the anterior commissure, and $4 \mathrm{~mm}$ inferior to the intercommissural line, with the trajectory of implantation through the ALIC (Fig. 2B). Stimulation contacts were shown in Figure 2C. Stimulation parameters of contact points 1 and 3 were set 1 month after operation, with a pulse width of $240 \mu \mathrm{s}$, a frequency of $150 \mathrm{~Hz}$, and a voltage of $2.5 \mathrm{~V}$ (Table 1). The patient still had auditory hallucination 6 months after operation, with improved mood and no suicidal thoughts. The scores of HAMA, HAMD, 
and PANSS were 11, 10 and 40 respectively. The parameters of the contact point 1 were adjusted to $240 \mu \mathrm{s}, 160 \mathrm{~Hz}$ and $3.0 \mathrm{~V}$ for considering that she still had auditory hallucination. Unfortunately, the symptom of auditory hallucination went worse, so the contact point 1 was given down to $60 \mu \mathrm{s}, 90 \mathrm{~Hz}$ and $1.0 \mathrm{~V}$. The auditory hallucination was alleviated, but anxiety and depression were aggravated 12 months after operation. The scores of HAMA, HAMD, and PANSS were 15, 16, and 36 respectively. Therefore, the parameters of the contact point 3 were adjusted to $240 \mu \mathrm{s}, 160 \mathrm{~Hz}$ and $2.5 \mathrm{~V}$. The patient's condition was stable 18 months after operation. All the psychiatric symptoms were improved, including auditory hallucination, anxiety, and depression. The scores of HAMA, HAMD, and PANSS were 7, 7 and 34 respectively. Head MRS showed levels of Cho, creatine (Cr), Cr2 and $\mathrm{N}$-acetyl aspartate were normal (Fig. 3C, 3D).

This study was approved by the ethics committee of Brain Hospital of Hunan Province (NO. K2017012). A written informed consent was obtained from the patients for the publication of this case report.

\section{DISCUSSION}

The patient suffered from auditory hallucination, insomnia, anxiety, poor mood and suicidal behavior after TBI. She tried a variety of antipsychotic drugs but they didn't work, so the DBS operation was adopted. Shin et al. [11] declared DBS could ameliorate motor, cognitive and emotional disorders after TBI, but they didn't discuss how to choose the target if psychiatric symptoms occurred. The patient had hallucination and emotional changes. Mikell et al. [12] considered that chronic, high-frequency electrical stimulation to the hippocampus or NAc may improve positive symptoms of schizophrenia via stabilizing the dopamine release in the striatum. The human NAc locates among the caudate nucleus, ventral side of the forelimb of the internal capsule and uncinate fasciculus. The NAc and the olfactory tubercle collectively form the ventral striatum, and it's also a part of the basal nucleus. We performed a head MRS scan to the patient and found elevated choline in the bilateral basal ganglia. It has been reported that abnormal choline metabolism was associated with illusion in schizophrenia; dopamine and choline may affect each other in the pathogenesis of mental disorders [13]. The $M$ receptor agonists could induce dop- amine release [14]. It can be explained that the abnormal choline metabolism of the NAC and the ALIC may be related to the mental abnormality of the patient.

Right now, more and more DBS operations are targeting ALIC-NAC in mental disorders, such as addiction, obsessive and depression [15-17]. Scholars evaluated the effects of DBS in the NAc core on the extracellular concentration of monoaminergic neurotransmitters in the medial prefrontal cortex and orbitofrontal cortex, and found rapid increases in the release of dopamine, serotonin and noradrenaline [18]. Thus we assumed perhaps stimulation to ALIC-NAC will improve hallucinations. This patient's mood symptoms were more serious than psychiatric symptoms, so the ALIC and NAc were selected to be the DBS stimulation targets.

In this case, the patient had both emotional symptoms and hallucination, so the settings of target stimulation parameters were of great importance. Rauch et al. [19] believed that low-frequency stimulation of the nucleus played a similar role in activating the nucleus, while high-frequency stimulation above $100 \mathrm{~Hz}$ inhibited or blockaded the related nerve conduction pathways. Therefore, we used high frequency to stimulate the ALIC and NAc at the very beginning and found that the patient's mood improved but the auditory hallucination still existed. The stimulation frequency had to be modified. The final results demonstrated that the frequency above $150 \mathrm{~Hz}$ aggravated auditory hallucination, while the frequency below $100 \mathrm{~Hz}$ improved it. Auditory hallucination improvement was mainly related to adjusting the stimulation frequency to NAc, and emotional relief required high-frequency stimulation to the ALIC. Only one patient was included, which was the shortcoming of this study. Moreover, the patient is too old to carry out more examinations. The exact mechanism of TBI-induced psychiatric symptoms remains unclear and requires further study.

In conclusion, we declare that DBS is reversible, adjustable and safe in the treatment of TBI-induced psychiatric symptoms. DBS to the ALIC-NAC should be considered as a possible treatment choice once a patient showed psychiatric symptoms after TBI.

\section{- Acknowledgments}

This work was supported by grants from the Science\& Technology Innovation Project of Hunan Province (grant 
number 2017SK50317, 2017SK50313) and Foundation of Health and Family Planning Commission of Hunan Province (grant number 20190058, 20190308). The authors would like to thank the patient and their family members for their cooperation.

\section{- Conflicts of Interest}

No potential conflict of interest relevant to this article was reported.

\section{Author Contributions}

Design: Liang Li. Data collection: Ping Yang. Operation: Weiping Kuang, Hongxing Huang. Postoperative follow-up: Yong Zhu, Xiaofeng Chen. Writing-original draft: Bin Zhou. Writing - review \& editing: Ping Yang.

\section{口 ORCID}

Bin Zhou

Weiping Kuang

https://orcid.org/0000-0002-1504-030X

Hongxing Huang:

Yong Zhu

Xiaofeng Chen

Liang Li

Ping Yang https://orcid.org/0000-0002-0311-3516 https://orcid.org/0000-0001-5478-7724 https://orcid.org/0000-0003-3492-9056 https://orcid.org/0000-0002-0172-1227 https://orcid.org/0000-0001-9994-4690 https://orcid.org/0000-0003-2375-0616

\section{REFERENCES}

1. McGuire JL, Ngwenya LB, McCullumsmith RE. Neurotransmitter changes after traumatic brain injury: an update for new treatment strategies. Mol Psychiatry 2019;24:995-1012.

2. Auxéméry Y. Mild traumatic brain injury and postconcussive syndrome: a re-emergent questioning. Encephale 2012;38: 329-335.

3. Awwad HO, Durand CD, Gonzalez LP, Tompkins P, Zhang Y, Lerner MR, et al. Post-blast treatment with Nociceptin/Orphanin FQ peptide (NOP) receptor antagonist reduces brain injuryinduced hypoxia and signaling proteins in vestibulomotor-related brain regions. Behav Brain Res 2018;340:183-194.

4. Domnick NK, Gretenkord S, De Feo V, Sedlacik J, Brockmann MD, Hanganu-Opatz IL. Neonatal hypoxia-ischemia impairs juvenile recognition memory by disrupting the maturation of prefrontal-hippocampal networks. Exp Neurol 2015;273: 202-214.

5. Albrecht JS, Mullins DC, Smith GS, Rao V. Psychotropic medication use among medicare beneficiaries following traumatic brain injury. Am J Geriatr Psychiatry 2017;25:415-424.

6. Testini P, Min HK, Bashir A, Lee KH. Deep brain stimulation for Tourette's syndrome: the case for targeting the thalamic centromedian-parafascicular complex. Front Neurol 2016;7: 193.

7. Hirschtritt ME, Bloch MH, Mathews CA. Deep brain stimulation for obsessive-compulsive disorder-reply. JAMA 2017; 318:393.

8. Hamilton M. The assessment of anxiety states by rating. $\mathrm{Br} J$ Med Psychol 1959;32:50-55.

9. Hamilton M. A rating scale for depression. J Neurol Neurosurg Psychiatry 1960;23:56-62.

10. Park JH, Hong JS, Kim SM, Min KJ, Chung US, Han DH. Effects of amisulpride adjunctive therapy on working memory and brain metabolism in the frontal cortex of patients with schizophrenia: a preliminary positron emission tomography/computerized tomography investigation. Clin Psychopharmacol Neurosci 2019;17:250-260.

11. Shin SS, Dixon CE, Okonkwo DO, Richardson RM. Neurostimulation for traumatic brain injury I Neurosurg 2014; 121:1219-1231.

12. Mikell CB, McKhann GM, Segal S, McGovern RA, Wallenstein $\mathrm{MB}, \mathrm{Moore} \mathrm{H}$. The hippocampus and nucleus accumbens as potential therapeutic targets for neurosurgical intervention in schizophrenia. Stereotact Funct Neurosurg 2009;87:256-265.

13. Martínez-Granados B, Brotons O, Martínez-Bisbal MC, Celda B, Martí-Bonmati L, Aguilar EJ, et al. Spectroscopic metabolomic abnormalities in the thalamus related to auditory hallucinations in patients with schizophrenia. Schizophr Res 2008;104:13-22.

14. Mirza NR, Peters D, Sparks RG. Xanomeline and the antipsychotic potential of muscarinic receptor subtype selective agonists. CNS Drug Rev 2003;9:159-186.

15. Huys D, Kohl S, Baldermann JC, Timmermann L, Sturm V, Visser-Vandewalle V, et al. Open-label trial of anterior limb of internal capsule-nucleus accumbens deep brain stimulation for obsessive-compulsive disorder: insights gained. I Neurol Neurosurg Psychiatry 2019;90:805-812.

16. Qu L, Ge S, Li N, Wang W, Yang K, Wu P, et al. Clinical evaluation of deep brain stimulation of nucleus accumbens/anterior limb of internal capsule for opioid relapse prevention: protocol of a multicentre, prospective and double-blinded study. BMJ Open 2019;9:e023516.

17. Schlaepfer TE, Bewernick BH. Deep brain stimulation for major depression. Handb Clin Neurol 2013;116:235-243.

18. van Dijk A, Klompmakers AA, Feenstra MG, Denys D. Deep brain stimulation of the accumbens increases dopamine, serotonin, and noradrenaline in the prefrontal cortex. J Neurochem 2012;123:897-903.

19. Rauch SL, Dougherty DD, Malone D, Rezai A, Friehs G, Fischman AJ, et al. A functional neuroimaging investigation of deep brain stimulation in patients with obsessive-compulsive disorder. J Neurosurg 2006;104:558-565. 